\title{
Use of Ground Planes within the Spatial Images Technique: Application to the Analysis of Rectangular Multilayered Shielded Enclosures
}

\author{
J. S. Gomez-Diaz, M. Garcia-Vigueras, D. Cañete-Rebenaque, F. D. Quesada-Pereira and A. Álvarez-Melcón \\ Technical University of Cartagena, Antiguo Cuartel de Antigones \\ Campus Muralla del Mar s/n; 30202 Cartagena, Murcia, Spain. \\ E-mail: jsebastian_gomez@ono.com, alejandro.alvarez@upct.es
}

\begin{abstract}
The use of ground planes is proposed inside the spatial images technique in order to calculate the multilayered shielded Green's functions for rectangular enclosures. The positions of the ground planes are dynamically located, covering two walls of the cavity, as a function of the source position. Spatial mirror images, related to the ground planes, are used to perfectly impose the boundary conditions along these cavity walls. This completely removes the problems associated to the singular behavior of the source when it is placed close to a wall or a corner, improving numerical stability. In addition, the method leads to a very efficient computation (about 15 times faster than the original approach), due to the reduced number of non-mirror images that must be effectively computed. A multi-band filter in hybrid waveguide-microstrip technology is analyzed with the new method, showing excellent agreement with measured data.
\end{abstract}

\section{INTRODUCTION}

The spatial images technique has recently been developed for the Green's functions computation, inside circular [1] and complex shaped convex [2], [3] multilayered cavities. However, some convergence problems occur when the source point is situated close to the cavity wall, due to the presence of spatial images with strong singular behavior. To solve this problem, optimization techniques [2] can be employed. Although these techniques are accurate, they considerably increase the computational times when modeling practical devices.

In this paper, we propose the combination of ground planes with the spatial images technique for the analysis of multilayer rectangular cavities. The ground planes are placed covering two of the cavity walls, as a function of the source position. Mirror charge/dipole images are then placed with respect to them. The value of these images is well-known from basic electromagnetic theory [4], and the effective number of images that need to be numerically calculated is reduced. The combinations of all images perfectly impose the potential boundary conditions on the covered cavity walls, while these conditions are numerically imposed on the remaining two walls (as in the previous approach [3]). Note that, following this technique, the convergence problems when the source point is close to a cavity wall or corner are avoided. This can be done by placing the ground planes at the two closest cavity walls to the source planes. In this way, the boundary conditions will automatically be imposed at these critical planes. This completely removes the problems associated to the singular behavior of the source when it is placed close to a wall or a corner, improving numerical stability. By following this strategy, the positions of the ground planes can be dynamically changed, according to the position of the source point inside the cavity. In this way, accuracy is preserved for all positions of the source point.

The importance of the method proposed is two fold. First, the boundary conditions are exactly satisfied along the cavity walls located close to the source point (which otherwise are prone to present numerical instabilities). Second, the computational cost is reduced, due to the fact that less number of images need to be numerically computed during the calculation of the final cavity Green's functions. In fact, although more mirror images appear, their values are already known from basic image theory [4], so the size of the linear system of equations to solve (see [3]) is effectively reduced.

Finally, to demonstrate the usefulness of the developed method, a practical multi-band filter in hybrid waveguidemicrostrip technology is analyzed. The filter combines two printed lines microstrip resonances with a cavity resonance, leading to a novel design. The proposed method agrees very well with respect to measurements, and it is more than 15 times faster than the original "completely numerical" approach [3].

\section{TheOreticAl OVERVIEW}

Let us consider a rectangular multilayered cavity, which is excited by a point source. The proposed implementation of the spatial images technique uses two auxiliary ground planes, which are located along two of the perfect electric walls of the cavity under analysis. As previously commented, the position of these planes can dynamically be changed, as a function of the point source position.

The main advantage of using ground planes placed along the cavity walls, is that the boundary conditions along these walls are automatically imposed by the set of spatial images. Consequently, the boundary conditions need to be enforced only on the remaining walls. This considerably reduces the number of unknowns during the numerical treatment of the problem. When the ground planes are placed, several mirror images, of known values, appear in the global image set. This 


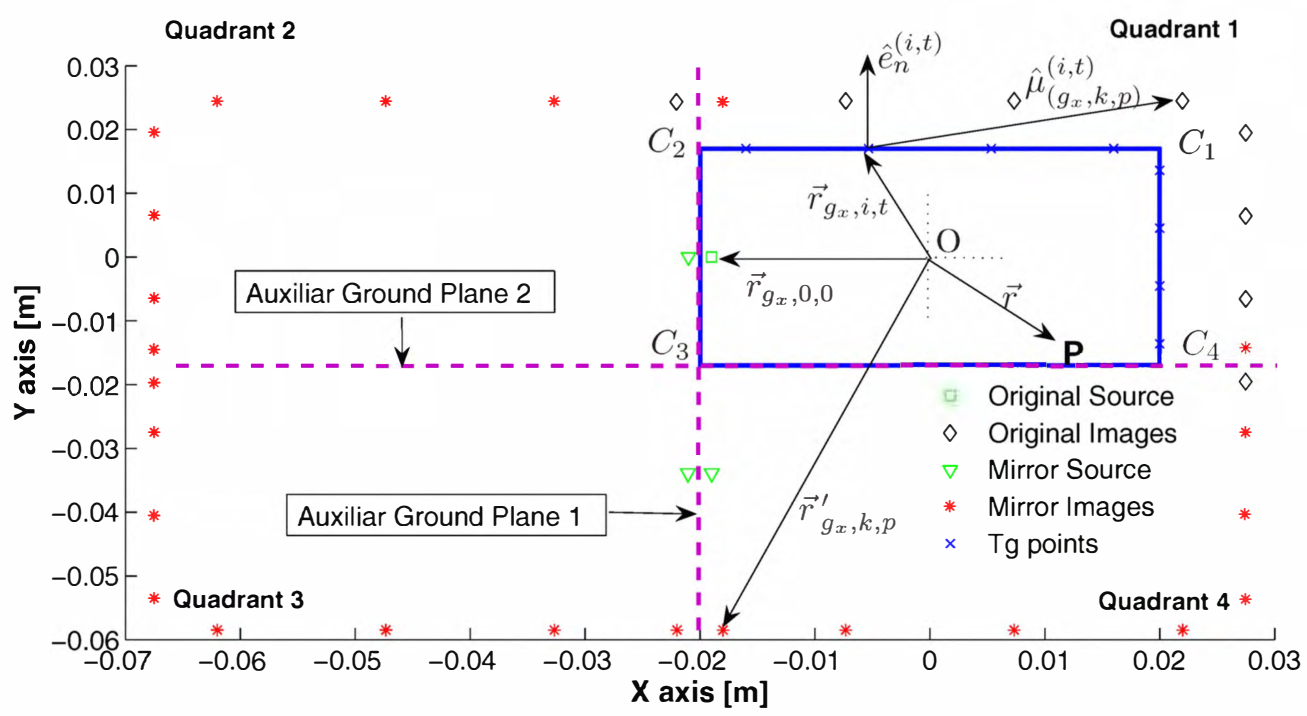

Fig. 1. Use of two auxiliary ground planes inside the spatial images technique, to analyze a multilayered rectangular enclosure. Spatial mirror images, with respect to the ground planes, appear from the original images. Potential boundary conditions are numerically imposed at discrete points ("Tg points") along the non-covered cavity walls, and are perfectly imposed along the covered walls. The dimensions of the cavity are $40 x 34$ mm, and is composed of 2 layers: a dielectric layer $\left(\epsilon_{r}=2.2\right.$ of thickness $\left.3.17 \mathrm{~mm}\right)$, and an air layer $(3.0 \mathrm{~mm}$ height). The source is placed at the position $(-19,0.0,3.14)$ mm. $O$ is the coordinates origin and cavity center, and $P$ is an observation point inside the cavity.

is represented, for the case of a rectangular multilayered cavity, in Fig. 1.

Specifically, the boundary conditions for the potentials are imposed at $N \times M$ discrete points along two walls of the cavity, distributed in $M$ rings of $N$ images. These walls are those not covered by the ground planes (see Fig. 1). Then, $N \mathrm{x} M$ spatial images are used to impose the boundary conditions, as in the regular implementation of this technique. However, due to the presence of ground planes, mirror images appear with respect to them. These mirror images are related to both, the original spatial images and the point source. Therefore, the total number of spatial images is now $4 \times N \times M+3$. Note that all mirror images will take the same values as the original images, but with different signs, according to standard image theory [4]. Finally, the interaction of all images and the source provides an exact fulfillment of the boundary conditions along the cavity walls where the ground planes are located, and an excellent numerical approximation on the two remaining planes.

The calculation of the electric scalar potential requires the solution of the following system of linear equations:

$$
\begin{aligned}
& \sum_{p=1}^{M} \sum_{k=1}^{N} q_{k, p} A\left(\vec{r}_{i, t}, \vec{r}_{k, p}^{\prime}\right)=-A\left(\vec{r}_{i, t}, \vec{r}_{0,0}^{\prime}\right) \\
& t=1,2,3, \ldots, M ; \quad i=1,2,3, \ldots, N
\end{aligned}
$$

where $A$ is defined as

$$
\begin{aligned}
A\left(\vec{r}, \vec{r}_{k, p}^{\prime}\right) & =S_{0}\left[\tilde{G}_{V}\left(\vec{r}, \vec{r}_{g_{1}, k, p}^{\prime}\right)\right]-S_{0}\left[\tilde{G}_{V}\left(\vec{r}, \vec{r}_{g_{2}, k, p}^{\prime}\right)\right] \\
& +S_{0}\left[\tilde{G}_{V}\left(\vec{r}, \vec{r}_{g_{3}, k, p}^{\prime}\right)\right]-S_{0}\left[\tilde{G}_{V}\left(\vec{r}, \vec{r}_{g_{4}, k, p}^{\prime}\right)\right]
\end{aligned}
$$

With the above notation, we refer to the $k$-th image inside the $p$-th ring and placed in the $g_{x}$ quadrant $(x=1,2,3,4)$, using the position vector: $\vec{r}_{g_{x}, k, p}^{\prime}$. Note that the quadrant (1) contains the original spatial images, while all other quadrants contain the mirror images with respect to the two ground planes. In a similar way, for the imposition of the boundary conditions we refer to the $i$-th point along the cavity wall inside the $t$-th ring, with the position vector: $\vec{r}_{i, t}$. It is important to note that these points are placed only along the two cavity walls not covered by the two ground planes. This is because the boundary conditions will automatically be imposed along the cavity walls where the ground planes are placed. Finally, the source position is denoted with the position vector $\vec{r}_{0,0}{ }^{\prime}$. From the previous equation, it is interesting to observe that the calculation of the cavity Green's functions requires the evaluation of the Green's functions due to the isolated images, placed inside an infinite multilayered medium. This is simply done through the use of the Sommerfeld transformation of order zero $\left(S_{0}\right)$ [5], applied to the corresponding spectral domain multilayered media Green's function $\left(\tilde{G}_{V}\right)$. The solution of the system of Eq. 1 provides the unknown complex charges $\left(q_{k, p}\right)$. The final electric scalar potential Green's function is then calculated with the following finite sum:

$$
G_{V_{T}}(\vec{r})=A\left(\vec{r}, \vec{r}_{0,0}^{\prime}\right)+\sum_{p=1}^{M} \sum_{k=1}^{N} q_{k, p} A\left(\vec{r}, \vec{r}_{k, p}^{\prime}\right)
$$

For the evaluation of the magnetic vector potential dyadic Green's functions, a similar procedure is followed. When the source dipole is oriented along the $x$-axis, the following system 
of linear equations is obtained:

$$
\begin{aligned}
- & \sin \varphi_{i, t} \sum_{p=1}^{M} \sum_{k=1}^{N} T\left(\vec{r}, \vec{r}_{k, p}^{\prime}\right) I_{k, p}^{x}+ \\
\cos \varphi_{i, t} & \sum_{p=1}^{M} \sum_{k=1}^{N} U\left(\vec{r}, \vec{r}_{k, p}^{\prime}\right) I_{k, p}^{y}=\sin \varphi_{i, t} T\left(\vec{r}_{i, t}, \vec{r}_{0,0}^{\prime}\right) \\
\cos \varphi_{i, t} & \sum_{p=1}^{M} \sum_{k=1}^{N} V_{(k, p)}^{(i, t)} I_{k, p}^{x}+ \\
\sin \varphi_{i, t} & \sum_{p=1}^{M} \sum_{k=1}^{N} W_{(k, p)}^{(i, t)} I_{k, p}^{y}=-\cos \varphi_{i, t} V_{(0,0)}^{(i, t)} \\
t & =1,2,3, \ldots, M \quad i=1,2,3, \ldots, N
\end{aligned}
$$

where $T, U, V$ and $W$ are defined as

$$
\begin{aligned}
T\left(\vec{r}, \vec{r}_{k, p}^{\prime}\right)= & S_{0}\left[\tilde{G}_{A}\left(\vec{r}, \vec{r}_{g_{1}, k, p}^{\prime}\right)\right]+S_{0}\left[\tilde{G}_{A}\left(\vec{r}, \vec{r}_{g_{2}, k, p}^{\prime}\right)\right] \\
- & S_{0}\left[\tilde{G}_{A}\left(\vec{r}, \vec{r}_{g_{3}, k, p}^{\prime}\right)\right]-S_{0}\left[\tilde{G}_{A}\left(\vec{r}, \vec{r}_{g_{4}, k, p}^{\prime}\right)\right] \\
U\left(\vec{r}, \vec{r}_{k, p}^{\prime}\right)= & S_{0}\left[\tilde{G}_{A}\left(\vec{r}, \vec{r}_{g_{1}, k, p}^{\prime}\right)\right]-S_{0}\left[\tilde{G}_{A}\left(\vec{r}, \vec{r}_{g_{2}, k, p}^{\prime}\right)\right] \\
- & S_{0}\left[\tilde{G}_{A}\left(\vec{r}, \vec{r}_{g_{3}, k, p}^{\prime}\right)\right]+S_{0}\left[\tilde{G}_{A}\left(\vec{r}, \vec{r}_{g_{4}, k, p}^{\prime}\right)\right] \\
V_{(k, p)}^{(i, t)}= & -S_{1}\left[\tilde{G}_{A}\left(\vec{r}_{i, t}, \vec{r}_{g_{1}, k, p}^{\prime}\right)\right] \hat{e}_{n}^{(i, t)} \cdot \hat{\mu}_{\left(g_{1}, k, p\right)}^{(i, t)} \\
& -S_{1}\left[\tilde{G}_{A}\left(\vec{r}_{i, t}, \vec{r}_{g_{2}, k, p}^{\prime}\right)\right] \hat{e}_{n}^{(i, t)} \cdot \hat{\mu}_{\left(g_{2}, k, p\right)}^{(i, t)} \\
& +S_{1}\left[\tilde{G}_{A}\left(\vec{r}_{i, t}, \vec{r}_{g_{3}, k, p}^{\prime}\right)\right] \hat{e}_{n}^{(i, t)} \cdot \hat{\mu}_{\left(g_{3}, k, p\right)}^{(i, t)} \\
& +S_{1}\left[\tilde{G}_{A}\left(\vec{r}_{i, t}, \vec{r}_{g_{4}, k, p}^{\prime}\right)\right] \hat{e}_{n}^{(i, t)} \cdot \hat{\mu}_{\left(g_{4}, k, p\right)}^{(i, t)}, \\
& \\
W_{(k, p)}^{(i, t)}= & -S_{1}\left[\tilde{G}_{A}\left(\vec{r}_{i, t}, \vec{r}_{g_{1}, k, p}^{\prime}\right)\right] \hat{e}_{n}^{(i, t)} \cdot \hat{\mu}_{\left(g_{1}, k, p\right)}^{(i, t)} \\
& +S_{1}\left[\tilde{G}_{A}\left(\vec{r}_{i, t}, \vec{r}_{g_{2}, k, p}^{\prime}\right)\right] \hat{e}_{n}^{(i, t)} \cdot \hat{\mu}_{\left(g_{2}, k, p\right)}^{(i, t)} \\
& +S_{1}\left[\tilde{G}_{A}\left(\vec{r}_{i, t}, \vec{r}_{g_{3}, k, p}^{\prime}\right)\right] \hat{e}_{n}^{(i, t)} \cdot \hat{\mu}_{\left(g_{3}, k, p\right)}^{(i, t)} \\
& -S_{1}\left[\tilde{G}_{A}\left(\vec{r}_{i, t}, \vec{r}_{g_{4}, k, p}^{\prime}\right)\right] \hat{e}_{n}^{(i, t)} \cdot \hat{\mu}_{\left(g_{4}, k, p\right)}^{(i, t)} .
\end{aligned}
$$

It is important to note the different vectors and angles employed in the formulation (see Fig. 1). In particular, we calculate the scalar product between the unitary vector $\hat{e}_{n}^{i, t}$ [normal to the wall where the point $(i, t)$ is defined] and the unitary vector $\left[\hat{\mu}_{\left(g_{x}, k, p\right)}^{(i, t)}\right]$, which relates the $\left(g_{x}, k, p\right)$ image with the $(i, t)$ point. In addition, $\varphi_{i, t}$ is defined as the angle between the unitary vector $\left(\hat{e}_{n}^{i, t}\right)$ and the $x$-axis. In the case of rectangular enclosures, $\varphi_{i, t}$ is equal to 0 or to $\pi / 2$ for $x$ and $y$-oriented cavity walls, respectively. It is also important to remark that in this case a Sommerfeld transformation of first order $\left(S_{1}\right)$ [5] is involved in equations (5c)-(5d). This is due to the spatial derivatives needed during the imposition of the boundary conditions for the field components normal to the cavity walls.

The solution of this system of equations gives the unknown complex amplitudes of all $x$-directed $\left(I_{k, p}^{x}\right)$ and $y$-directed
$\left(I_{k, p}^{y}\right)$ dipole images. Once they are found, the corresponding dyadic components of the magnetic vector potential are recovered with the following finite sums:

$$
\begin{aligned}
G_{A_{T}}^{x x}\left(\vec{r}, \vec{r}_{0}^{\prime}\right) & =T\left(\vec{r}, \vec{r}_{0,0}^{\prime}\right)+\sum_{p=1}^{M} \sum_{k=1}^{N} I_{k, p}^{x} T\left(\vec{r}, \vec{r}_{k, p}^{\prime}\right) \\
G_{A_{T}}^{y x}\left(\vec{r}, \vec{r}_{0}^{\prime}\right) & =\sum_{p=1}^{M} \sum_{k=1}^{N} I_{k, p}^{y} U\left(\vec{r}, \vec{r}_{k, p}^{\prime}\right)
\end{aligned}
$$

In the case that the source dipole is placed along the $y$-axis, similar expressions can be obtained.

The location of the spatial images around the cavity under analysis has always been an important degree of freedom of this technique, with direct impact in the accuracy of the method. In contrast to other optimization methods [2], the method proposed in this paper imposes a perfect fulfillment of the boundary conditions in two walls, removing the problems associated to the singular behavior of the source when it is placed close to a wall or a corner. Furthermore, the ground planes can dynamically be selected as a function of the point source position inside the cavity, enforcing the boundary conditions at the cavity walls closest to the source. Therefore, since the spatial images do not have to compensate numerically for the singular behavior of the source [3] (this is done automatically by the ground planes), the impact of the images positions in the accuracy of the technique is greatly reduced.

Another interesting remark is the reduction of the time required to compute the Green's functions. Although the number of total images increases with the new method, most of them are exact mirror images. In fact, the number of images values that need to be numerically computed is reduced, so the time to solve the three required linear systems for each source drastically decreases. This reduction is a direct consequence that the boundary conditions are automatically imposed by the selected set of images, at two of the walls of the cavity.

\section{RESULTS}

In this section, we will first demonstrate that the proposed method is able to accurately compute the Green's Functions, even when the source is placed extremely close to a cavity wall (see Fig. 1). For this purpose, Fig. 2 represents the electric scalar potential along the perimeter contour of the cavity, obtained with the original and with the new proposed technique based on ground planes. An ideal situation would provide zero value of the electric scalar potential along the cavity contour. As it can be observed in the figure, the new method obtains exact results along the walls where the ground planes are placed. For the other two walls, the potential exhibits a typical low-value ripple, showing zero values at the points where the boundary conditions are enforced. Note that the original method can not compensate for the source singular behavior by using the set of images. This problems has totally been solved with the proposed method.

The computed Green's functions have been employed for the analysis of a practical dual-band filter in hybrid waveguidemicrostrip technology, printed inside a rectangular cavity [6]. 


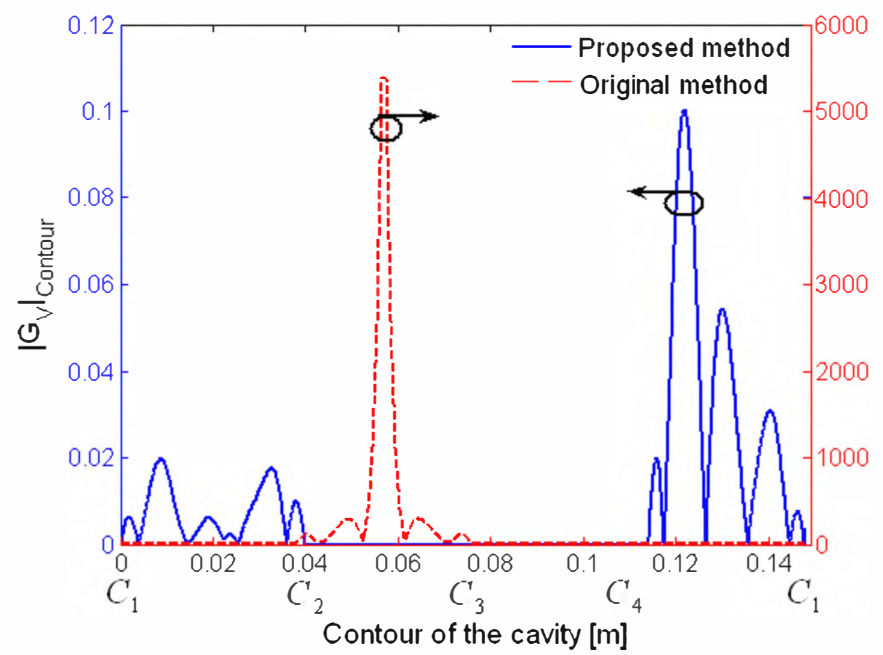

Fig. 2. Electric scalar potential $\left|G_{V}\right|$ along the rectangular cavity contour (see Fig. 1), evaluated with the proposed method and with the original spatial images technique. The source is placed close to the wall at the position $(-19,0.0,3.14) \mathrm{mm}$. The labels $C_{1}, C_{2}, C_{3}$ and $C_{4}$ correspond to the corners of the cavity shown in Fig. 1.

The device is sketched in Fig. 3. This filter combines two mi-

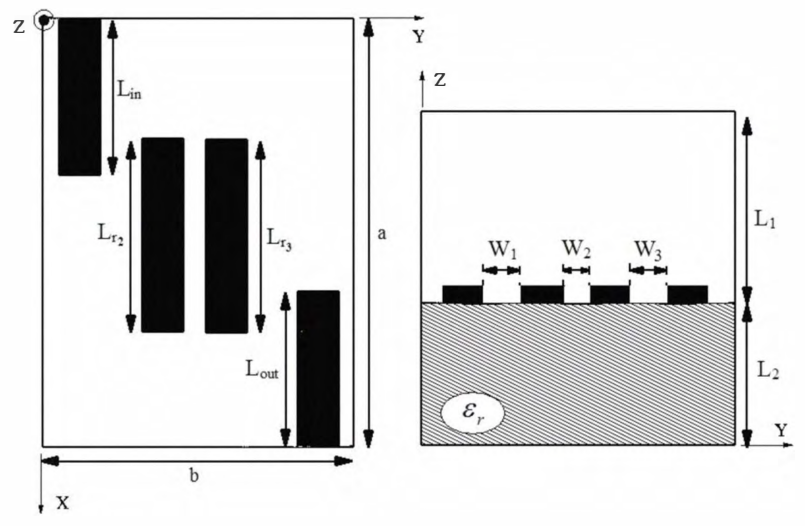

Fig. 3. Multi-band filter in hybrid waveguide-microstrip technology. $L_{1}=$ $3.0 \mathrm{~mm}, L_{2}=3.15 \mathrm{~mm}, \epsilon_{r}=2.2 \mathrm{~mm}, L_{\text {in }}=L_{\text {out }}=14.0 \mathrm{~mm}, L_{r 1}=$ $L_{r 2}=24.54 \mathrm{~mm}, W_{1}=W_{3}=1.8 \mathrm{~mm}, W_{2}=5.0 \mathrm{~mm}, a=40.0 \mathrm{~mm}$, $b=34.0 \mathrm{~mm}$.

crostrip resonances with a cavity resonance in order to obtain a third order dual-bandpass filter. It is important to remark that the correct model of the partially filled cavity is fundamental in this case, due to the fact that it provides one of the resonances of the filter. The results of the analysis employing the new method based on ground planes are presented in Fig. 4. Fullwave results [6] directly superimpose, and are not included here. Measured data is employed as validation, showing good agreement with simulated results.

Finally, note that the computational cost obtained with the technique just derived is very low. The analysis of the filter has taken 0.45 seconds per frequency point, whereas the original spatial images technique required 7.1 seconds per frequency point, i.e. the new technique is about 15 times faster.

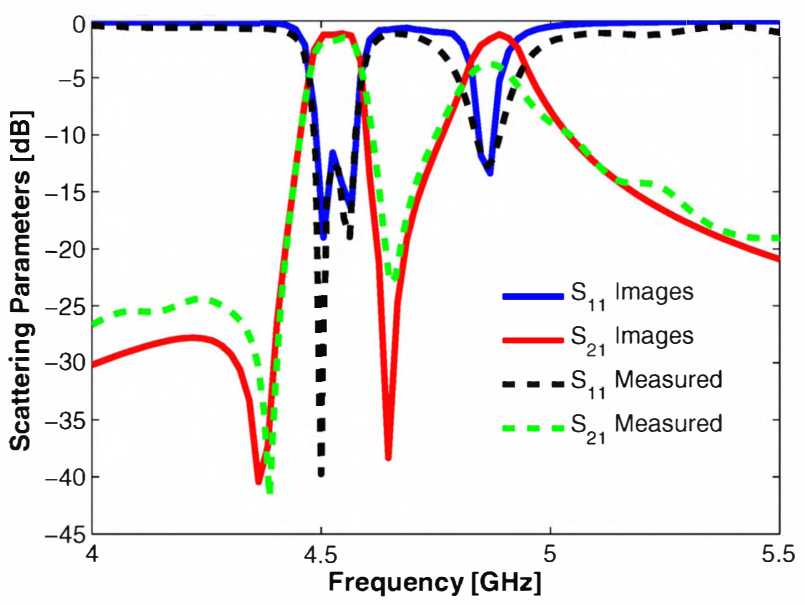

Fig. 4. Scattering parameter of the filter sketched in Fig. 3, analyzed with the new method proposed. Measured data is used as validation.

\section{CONClusions}

In this paper, ground planes have been employed to improve the spatial images technique, in order to calculate the multilayered shielded Green's functions related to rectangular cavities. The potential boundary conditions on the walls covered by the ground planes are exactly satisfied, thanks to the use of mirror spatial images. This improves the numerical stability of the method, removing the problems associated to the singular behavior of the source when it is placed close to a wall or a corner. In addition, the method leads to a very efficient computation (more than 15 times faster than the original), due to the reduced number of images that must be effectively computed. A multi-band filter in hybrid waveguide-microstrip technology has been analyzed with the new method, showing excellent agreement with measured data, and demonstrating the practical value of the technique.

\section{REFERENCES}

[1] F. Q. Pereira, P. V. Castejon, D. C. Rebenaque, J. P. Garcia, and A. A. Melcon, "Numerical evaluation of the Green's functions for cylindrical enclosures," IEEE Transactions on Microwave Theory and Techniques, vol. 53, pp. 94-105, January 2005.

[2] J. S. Gómez-Díaz, F. D. Quesada-Pereira, J. Pascual-Garcia, and A. Alvarez-Melcon, "Numerical Evaluation of the Green's Functions for Arbitrarily-Shaped Cylindrical Enclosures and their Optimization by a new Spatial Images Method," Radio Science, vol. 42, pp. 1-16, October 2007.

[3] J. S. Gómez-Díaz, M. Martínez-Mendoza, F. Perez-Soler, F. D. QuesadaPereira, and A. Alvarez-Melcon, "Practical Implementation of the Spatial Images Technique for the Analysis of Shileded Multilayered Printed Circuits," IEEE Transactions on Microwave Theory and Techniques, vol. 56, pp. 131-141, January 2008.

[4] C. A. Balanis, Advanced Engineering Electromagnetics. John Wiley and Sons, 1989.

[5] K. Michalski, "Extrapolation methods for Sommerfeld integral tails," IEEE Transactions on Antennas and Propagation, vol. 46, pp. 14051418, October 1998.

[6] M. Martínez-Mendoza, J. S. Gómez-Díaz, D. Canete-Rebenaque, and A. Alvarez-Melcon, "Design of Dual-Bandpass Hybrid WaveguideMicrostrip Microwave Filters," IEEE Transactions on Microwave Theory and Techniques, vol. 56, pp. 2913-2920, December 2008. 